\title{
MINERALIZAÇÃO DE COMPOSTOS NITROGENADOS DE LODOS DE ESGOTO NA QUINTA APLICAÇÃO EM LATOSSOLO $^{(1)}$
}

\author{
Rita Carla Boeira ${ }^{(2)}$ \& Viviane Cristina Bettanin Maximiliano ${ }^{(3)}$
}

\begin{abstract}
RESUMO
Em sistemas agrícolas em que se aplica lodo de esgoto, devem ser consideradas as necessidades de $\mathrm{N}$ das plantas e, simultaneamente, evitar a geração de excesso de nitrato, poluente potencial de águas subsuperficiais. Para isso, na determinação das quantidades máximas a serem aplicadas em determinado cultivo, devem-se conhecer algumas propriedades do lodo de esgoto e do solo. Uma delas é a fração de mineralização de compostos nitrogenados orgânicos (FMN) contidos nos resíduos que serão mineralizados durante o ciclo da cultura. Essa quantidade, somada ao $\mathrm{N}$ na forma mineral contido no lodo, fornece a quantidade de $\mathrm{N}$ do lodo de esgoto que ficará disponível durante a safra. Este trabalho teve como objetivo determinar se as aplicações anteriores de lodo de esgoto, feitas em um Latossolo, alteram a FMN do resíduo recentemente aplicado. $O$ solo foi previamente tratado, em quatro cultivos sucessivos de milho, com quatro doses e dois tipos de lodo de esgoto, um de origem urbana (Franca, SP) e outro de origem urbano-industrial (Barueri, SP). O experimento de mineralização foi instalado em laboratório, com delineamento experimental em blocos casualizados e três repetições, realizado durante 15 semanas. As doses dos lodos de esgoto estudadas, na quinta aplicação, corresponderam a 160, 320, 640 e $1.280 \mathrm{mg} \mathrm{kg}^{-1}$ de $\mathrm{N}$ orgânico. Em sete épocas, foram determinados o pH e os teores de $\mathrm{N}$ nas formas amoniacal e nítrica. A FMN foi calculada com os dados obtidos para a mineralização líquida medida 105 dias após o início da incubação. Os comportamentos dos dois tipos de lodo foram similares, com taxa de mineralização inicial elevada. Os teores de amônio mantiveram-se baixos logo após o início da incubação até o final, com aumento linear nos teores de nitrato. Houve maior acúmulo de nitrato para as maiores doses aplicadas. O potencial de mineralização dos tratamentos, estimado pelo modelo exponencial simples, variou entre 58 e $284 \mathrm{mg} \mathrm{kg}^{-1} \mathrm{de} \mathrm{N}$. Não houve efeito das quantidades de resíduos previamente aplicadas ao Latossolo sobre a
\end{abstract}

\footnotetext{
${ }^{(1)}$ Recebido para publicação em abril de 2008 e aprovado em fevereiro de 2009.

(2) Pesquisadora da Embrapa Meio Ambiente. Caixa Postal 69, CEP 13.820-000 Jaguariúna (SP). E-mail: rcboeira@cnpma.embrapa.br

${ }^{(3)}$ Assistente de Pesquisa da Embrapa Meio Ambiente. E-mail: viviane@cnpma.embrapa.br
} 
fração de mineralização do $\mathrm{N}$ orgânico recentemente aplicado via lodo de esgoto, com confiabilidade de $95 \%$. Os resultados mostraram que, em reaplicações na mesma área, as doses de lodo de esgoto devem ser menores do que as doses calculadas para aplicação única, devido ao $\mathrm{N}$ residual acumulado no solo, tanto na forma orgânica quanto inorgânica.

Termos de indexação: nitrato, amônio, reciclagem agrícola, $\mathrm{N}$ disponível, $\mathrm{pH}, \mathrm{N}$ potencialmente mineralizável.

\title{
SUMMARY: ORGANIC NITROGEN MINERALIZATION RATE FOLLOWING THE FIFTH SEWAGE SLUDGE APPLICATION IN AN OXISOL
}

\begin{abstract}
In agricultural systems treated with sewage sludge, the management ought to seek an adequate $N$ supply to plants, while avoiding nitrate enrichment because of its potential to pollute subsurface water bodies. Thus, when determining the maximum amounts to be applied to a specific crop, some sewage sludge and soil properties should be known. One is the fraction of organic $N$ of the waste that will be mineralized (NMF) during the crop cycle. This quantity, combined with mineral $N$ in the sludge, determines the amount of $N$ in sewage sludge that will be available during the growing season. This study aimed to determine whether previous applications of sewage sludge affect the NMF of the residue recently applied to a Dark Red Dystroferric Latosol (Oxisol). The soil had been previously treated in four successive maize crops, with four rates and two types of sewage sludge, one of urban origin (Franca, SP) and the other from urban-industrial sources (Barueri, SP). The study of $N$ mineralization was installed in laboratory in a randomized blocks design with three replications and lasted 15 weeks. In the fifth application, sewage sludge doses of equivalent to $160,320,640$, and 1,280 $\mathrm{mg} \mathrm{kg}^{-1}$ organic $N$ were studied. On seven dates, the $p H$ and the ammonium- $N$ and nitrate- $N$ concentration were determined. The NMF was calculated with data obtained for the net mineralization measured 105 days after the start of incubation. The performance of the two sludge types was similar, with a high initial mineralization rate. The ammonium levels remained low after the start until the end of incubation and there was a linear increase in nitrate levels during the same period. Nitrate accumulation peaked at the highest sewage sludge dose applied. The potential for mineralization, estimated by the simple exponential model, varied from 58 to $284 \mathrm{mg} \mathrm{kg}^{-1} \mathrm{~N}$. No effect of previously applied waste on the mineralization fraction of recently applied organic $N$ by sewage sludge to the Oxisol was observed, with a reliability of $95 \%$. Results showed that for reapplications to the same area, sewage sludge rates must be lower than the doses calculated for a single application, due to accumulations of organic and inorganic- $N$ in the soil.
\end{abstract}

Index terms: nitrate, ammonium, agricultural recycling, available $N$, $p H$, potentially mineralizable $N$.

\section{INTRODUÇÃO}

Os lodo de esgoto são ricos em matéria orgânica, em nitrogênio, em fósforo e em alguns outros nutrientes de plantas, podendo ocasionar melhoria do condicionamento do solo. Por essa razão e pelo alto custo de descarte dos resíduos gerados, o uso agrícola de lodo de esgoto vem sendo difundido no Brasil à medida que aumenta o número de estações de tratamento, públicas ou privadas. Nos Estados Unidos e na Europa, 40 \% do total de biossólidos produzidos são lançados em aterros sanitários, e o uso agrícola encontrava-se, no final do século XX, em torno de 25 e $37 \%$ do total em cada local, respectivamente (Tsutiya, 2000).
Paralelamente, lodos de esgoto correspondem a uma fonte potencial de riscos ao ambiente e potencializam a proliferação de vetores de moléstias e organismos nocivos, podendo conter metais pesados, compostos orgânicos persistentes e patógenos (CONAMA, 2006). Tais resíduos possuem centenas de compostos orgânicos poluentes, como antibióticos, anticoncepcionais, cosméticos, hormônios, azeites, detergentes, antiinflamatórios, fármacos psiquiátricos, etc. (Ternes, 1998; Ternes et al., 1999; Tsutiya, 2001; Boyd et al., 2003; Carballa et al., 2005; Saito, 2007), o que levou Hettenback et al. (1998) a questionar se é seguro ou conveniente o uso agrícola de lodo de esgoto em qualquer sistema de produção de alimentos, orgânico ou não. Paraíba et al. (2006), considerando apenas 
um dos diversos poluentes orgânicos presentes nos lodos das estações de Barueri e de Suzano, ambas em São Paulo, mostraram que doses maiores do que $10.000 \mathrm{~kg} \mathrm{ha}^{-1}$ não deveriam ser usadas para o cultivo de frutíferas. Em manejo de solo com aplicações repetidas, pesquisas recentes têm mostrado que, entre os nutrientes, o P também deve ser motivo de preocupação ambiental do uso agrícola de lodo de esgoto (Shober \& Sims, 2003; Schroder et al., 2008).

Segundo Schroder et al. (2008), a aplicação de lodo de esgoto acima das taxas de recomendação agronômica baseadas em $\mathrm{N}$ devem ser evitadas. Quando aplicados em quantidades elevadas e, ou, em aplicações sucessivas, os lodo de esgoto podem gerar riscos de poluição ambiental por adição de excesso de $\mathrm{N}$ de formas orgânicas, que degradam microbiologicamente a nitrato, conforme constataram Dynia et al. (2006) e Oliveira et al. (2001) em solos brasileiros. As raízes absorvem apenas parte desse íon, que é muito móvel no solo, alcançando facilmente corpos d'água superficiais ou sub-superficiais. Se ingerido, sofre redução a nitrito no sistema digestivo, podendo intoxicar crianças e animais jovens (Pimentel, 1996).

Por essa razão, a aplicação, por anos sucessivos, de lodo de esgoto a solos traz grandes preocupações. Barbarick et al. (1996) publicaram o primeiro estudo de mineralização de lodo de esgoto com aplicações repetidas durante vários anos, em região com baixa pluviosidade. Esses autores verificaram que, após seis ou sete aplicações anuais de biossólidos urbanos em solos cultivados com trigo, apenas 2 a $9 \%$ do $\mathrm{N}$ aplicado foi removido pelos grãos, 35 a $54 \%$ permaneceu no solo como $\mathrm{N}$ residual e 38 a $63 \%$ foi perdido por desnitrificação, volatilização e lixiviação. O nitrato pode também contribuir significativamente para a salinização de solos agrícolas, particularmente em regiões com baixa pluviosidade (Forste, 1996). A disponibilidade de Né, provavelmente, um dos fatores mais restritivos quando se aplica ao solo, pela primeira vez, lodo de esgoto não contaminado com outras substâncias, orgânicas ou inorgânicas. Considerandose as quantidades médias de $\mathrm{N}$ recomendadas para a maioria das culturas e as quantidades médias de $\mathrm{N}$ disponíveis em lodos, as doses devem variar, de acordo com este critério, de 5 a 20 t ha $^{-1}$ (base seca) (Wallace \& Wallace, 1994). Na Europa, há regiões classificadas como vulneráveis a nitrato, e a regulamentação considera não somente doses, mas também épocas de aplicação de compostos orgânicos, buscando reduzir a poluição das águas com nitrato (CEC, 1991; Reino Unido, 1996). No Brasil, a norma para utilização agrícola de lodo de esgoto (CONAMA, 2006) é baseada na norma americana (USEPA, 1996), dado o pequeno número de pesquisas nas condições brasileiras que enfatizem efeitos ambientais. As frações de mineralização recomendadas para o estabelecimento de doses de lodo de esgoto são, então, aquelas determinadas para climas temperados, como $20 \%$ para a primeira aplicação ao solo de lodo de esgoto com digestão anaeróbia.
Como a determinação da taxa agronômica de aplicação é crítica na prevenção da lixiviação de nitrato, é importante o estudo da dinâmica de $\mathrm{N}$ de lodo de esgoto em condições tropicais, tendo-se em vista o ajustamento real das doses às necessidades das plantas cultivadas. Para isso, a maior dificuldade é a predição das taxas de mineralização de N. A fração de mineralização de $\mathrm{N}$ orgânico de lodo de esgoto varia conforme o tipo de solo (Garau et al., 1986) e a origem do material. Parker \& Sommers (1983), nos Estados Unidos, obtiveram $15 \%$ em 16 semanas de incubação, e Boeira et al. (2002), no Brasil, obtiveram mais de $30 \%$ em 15 semanas de incubação em lodo de esgoto digerido anaerobiamente, na primeira aplicação ao solo. Consequentemente, até $70 \%$ do $\mathrm{N}$ orgânico adicionado, via lodo de esgoto, pode permanecer no solo após a colheita. Se houver nova aplicação, a esse $\mathrm{N}$ orgânico remanescente se soma o que é adicionado na safra seguinte, e assim sucessivamente, originando efeitos residuais acumulativos que podem afetar a mineralização da aplicação seguinte. Fernandes et al. (2005) concluíram que aplicações repetidas do lodo de esgoto de Barueri provocaram o estabelecimento de novas condições biológicas no solo ao avaliarem diversos índices, como respiração basal, quociente metabólico, $\mathrm{C}$ e $\mathrm{N}$ da biomassa microbiana. Tais mudanças podem afetar a velocidade de liberação do $\mathrm{N}$ e, ou, a quantidade de $\mathrm{N}$, que será mineralizada numa nova aplicação de lodo de esgoto.

No Brasil, as estações de tratamento de esgoto começaram a ser construídas na última década. Devido ao intenso aumento na produção de lodo de esgoto e seu consequente uso agrícola, há necessidade de pesquisas que mostrem quais são os efeitos, a longo prazo, de aplicações consecutivas desses resíduos sobre as propriedades físicas, químicas ou biológicas do solo.

O objetivo deste trabalho foi determinar se a fração de mineralização de $\mathrm{N}$ de formas orgânicas de lodo de esgoto recentemente aplicado depende da quantidade aplicada antes em sistemas de manejo do solo com reaplicações sucessivas desses resíduos.

\section{MATERIAL E MÉTODOS}

\section{Manejo prévio do Latossolo estudado}

Para a composição dos tratamentos deste trabalho, utilizaram-se amostras de solo de um estudo que vem sendo desenvolvido no campo experimental da Embrapa Meio Ambiente, desde 1999. A área situase em Jaguariúna, SP, latitude $22^{\circ} 41$ ' sul, longitude $47^{\circ} 00$ ' oeste e altitude $570 \mathrm{~m}$, em Latossolo Vermelho distroférrico, textura argilosa (Typic Haplustox). Neste experimento, o delineamento experimental foi de blocos casualizados, avaliando-se tipos e doses de lodo de esgoto em parcelas subdivididas. Nas parcelas e subparcelas, foram alocados os tipos de lodo e suas doses, respectivamente. A área de cada subparcela 
foi de $10 \times 20 \mathrm{~m}$. O lodo de esgoto foi aplicado ao solo antes de cada plantio de milho, em quatro cultivos sucessivos, visando à avaliação do uso agrícola de lodo a longo prazo. Alguns resultados referentes ao impacto ambiental desse estudo são relatados em Vieira \& Cardoso (2003), Vieira et al. (2005), Fernandes et al. (2005), Dynia et al. (2006) e em Boeira \& Souza (2007). Esses autores apresentam dados obtidos tanto antes quanto depois das amostragens de solo feitas para este trabalho. Os lodo de esgoto utilizados foram provenientes das estações de tratamento de esgotos sanitários das cidades de Franca (de origem doméstica) e de Barueri (de origem urbano-industrial), ambas no Estado de São Paulo. O sistema de tratamento nas duas estações foi a digestão anaeróbia dos lodos ativados, sem cal. As doses aplicadas foram codificadas como F $1 \mathrm{~N}, \mathrm{~F} 2 \mathrm{~N}, \mathrm{~F} 4 \mathrm{~N}, \mathrm{~F} 8 \mathrm{~N}, \mathrm{~B} 1 \mathrm{~N}, \mathrm{~B} 2 \mathrm{~N}, \mathrm{~B} 4 \mathrm{~N}$ e B $8 \mathrm{~N}$, relativas às quantidades apresentadas no quadro 1 . No primeiro cultivo, $\mathrm{F} 1 \mathrm{~N}$ e $\mathrm{B} 1 \mathrm{~N}$ representaram as doses máximas recomendadas para o lodo de esgoto de Franca e de Barueri, respectivamente, calculadas segundo CETESB (1999), e as demais representaram seus múltiplos. Nos três cultivos seguintes, essas doses foram reaplicadas, sem considerar o efeito residual das aplicações anteriores; no quadro 1, as diferenças nas quantidades de lodo nos quatro cultivos são devidas a variações nas concentrações de $\mathrm{N}$ dos lotes de lodos. O lodo úmido foi distribuído a lanço antes de cada cultivo, e incorporado com enxada rotativa à camada arável do solo, três a quatro dias antes de cada semeadura. Os tratos culturais foram os padrões para milho, retirando-se restos culturais antes da aplicação do lodo. Após a segunda e terceira colheitas, foi feita a correção da acidez do solo com calcário dolomítico.

Quadro 1. Quantidades de lodo de esgoto aplicadas previamente ao Latossolo, no experimento de campo, em quatro cultivos de milho

\begin{tabular}{|c|c|c|c|c|}
\hline \multirow{2}{*}{ Dose } & \multicolumn{4}{|c|}{ Cultivo } \\
\hline & Primeiro & Segundo & Terceiro & Quarto \\
\hline & & kg h & $\cdot 1$ & \\
\hline $\mathrm{F}^{(1)} 1 \mathrm{~N}^{(2)}$ & $3.014^{(3)}$ & 3.504 & 3.766 & 4.432 \\
\hline $\mathrm{F} 2 \mathrm{~N}$ & 6.028 & 7.008 & 7.532 & 8.864 \\
\hline $\mathrm{F} 4 \mathrm{~N}$ & 12.056 & 14.016 & 15.064 & 17.728 \\
\hline F $8 \mathrm{~N}$ & 24.112 & 28.032 & 30.128 & 35.456 \\
\hline $\mathrm{B}^{(1)} 1 \mathrm{~N}$ & 8.095 & 3.995 & 5.315 & 5.295 \\
\hline B $2 \mathrm{~N}$ & 16.190 & 7.990 & 10.630 & 10.590 \\
\hline B $4 \mathrm{~N}$ & 32.380 & 15.980 & 21.260 & 21.180 \\
\hline B $8 \mathrm{~N}$ & 64.760 & 31.960 & 42.520 & 42.360 \\
\hline
\end{tabular}

(1) Lodo de esgoto de Franca ou de Barueri, SP. ${ }^{(2)} 1 \mathrm{~N}$ : corresponde à aplicação, via lodo de esgoto, da dose de $\mathrm{N}$ disponível recomendada para o milho no Latossolo; $2 \mathrm{~N}, 4 \mathrm{~N}$ e $8 \mathrm{~N}$ representam múltiplos dessa dose. ${ }^{(3)}$ Resultados expressos com base em massa de matéria seca.
A amostragem deste trabalho foi efetuada cinco meses após a quarta aplicação do lodo, e após a quarta colheita. Subparcelas representativas das doses aplicadas previamente ao Latossolo (F $1 \mathrm{~N}, \mathrm{~F} 2 \mathrm{~N}$, etc.) foram amostradas na profundidade de 0 a $20 \mathrm{~cm}$, com 10 amostras simples, selecionando-se subparcelas que apresentavam $\mathrm{pH}$ em água acima de 5,5. A seguir, as amostras foram secas ao ar e passadas em peneira com malha de $5 \mathrm{~mm}$, mantidas com teor de água em capacidade de campo até o início da mineralização.

\section{Experimento de mineralização em laboratório}

O experimento de incubação aeróbia foi realizado em laboratório em temperatura ambiente (variação entre 17 e $27^{\circ} \mathrm{C}$ ), em local sem iluminação. Utilizouse o delineamento experimental em blocos casualizados, com três repetições, estudando-se 20 tratamentos, em sete épocas de avaliação. Para a composição dos tratamentos apresentados no quadro 2 , aplicaram-se doses de $\mathrm{N}$ orgânico, via lodo de esgoto, ao Latossolo previamente tratado com quatro aplicações. A dose de $160 \mathrm{mg} \mathrm{kg}^{-1}$ de $\mathrm{N}$ orgânico (Quadro 2) foi equivalente à aplicação de $100 \mathrm{~kg} \mathrm{ha}^{-1}$ de $\mathrm{N}$ mineral, calculada segundo CETESB (1999), e as demais foram seus múltiplos. Os lodos de esgoto foram secos ao ar, e, na época da realização do experimento, foram analisados para determinação do teor de N (Quadro 3). Os tratamentos foram avaliados no início da incubação (0 dias) e após 14, 28, 42, 63, 84 e 105 dias, utilizandose a mesma parcela experimental para todas as amostragens de um mesmo tratamento e repetição (processo não destrutivo). As unidades experimentais constituíram-se de microcosmos com $500 \mathrm{~g}$ de solo, misturado periodicamente para eliminação de crostas e distribuição uniforme de água e de ar, e mantido em capacidade de campo por meio de pesagens, permitindose trocas gasosas. $\mathrm{O} \mathrm{pH}$ foi determinado em relação solo:água 1:2,5 (Camargo et al., 1986). Os teores de $\mathrm{N}$ - $\left(\mathrm{NO}_{3}{ }^{-}+\mathrm{NO}_{2}{ }^{-}\right)$e de N-NH${ }^{+}$foram determinados por extração com KCl $1 \mathrm{~mol} \mathrm{~L}^{-1}$ seguida de destilação em meio alcalino com liga de Devarda (Tedesco et al., 1995), expressando-se os resultados com base em massa de matéria seca.

No início e no final do experimento, foi determinado nos tratamentos o teor de N-total pelo método Kjeidahl segundo Tedesco et al. (1995). Calcularam-se os valores de $\mathrm{N}$ mineral acumulado em seis períodos (de 0 a 14 dias até 0 a 105 dias). Os dados foram transformados em logaritmo neperiano (exceto $\mathrm{pH}$ ), visando sanar problemas de falta de normalidade estatística.

Quando indicado pela análise de variância, utilizouse o teste de Tukey $(p \leq 0,01)$ para comparação conjunta de médias dos tratamentos dentro de cada dose prévia de lodo de esgoto, e foram feitas análises de correlação entre as quantidades aplicadas de $\mathrm{N}$ orgânico e de N mineralizado e de regressão linear das variáveis estudadas em função das doses aplicadas de $\mathrm{N}$ orgânico. Os dados de $\mathrm{N}$ mineralizado nos seis períodos de incubação foram ajustados ao modelo proposto por Stanford \& Smith (1972), descrito pela 
equação $\mathrm{Nm}=\mathrm{No}\left(1-\mathrm{e}^{-\mathrm{kt}}\right)$, em que $\mathrm{Nm}\left(\mathrm{mg} \mathrm{kg}^{-1}\right.$ de $\mathrm{N}$ no solo) representa o valor estimado de $\mathrm{N}$ mineral gerado e acumulado em dado tempo t de incubação (dias) e k representa a constante de primeira ordem da taxa de mineralização de $\mathrm{N}$ orgânico (dia $\left.{ }^{-1}\right)$.
Os parâmetros No (N orgânico potencialmente mineralizável no solo; $\mathrm{mg} \mathrm{kg}^{-1}$ de $\mathrm{N}$ no solo) e k foram estimados por análise de regressão não linear (Smith et al., 1980). Para cada dose prévia de lodo de esgoto (Quadro 2), a fração de mineralização do $\mathrm{N}$ de formas

Quadro 2. Tratamentos avaliados e doses equivalentes, por área, dos dois lodos de esgoto utilizados na quinta aplicação em Latossolo

\begin{tabular}{|c|c|c|c|c|c|c|}
\hline \multirow{3}{*}{ Tratamento } & \multicolumn{2}{|c|}{ Composição do tratamento } & & & & \\
\hline & \multirow{2}{*}{$\begin{array}{l}\text { Dose prévia de lodo de } \\
\text { esgoto da primeira à } \\
\text { quarta aplicação }^{(1)}\end{array}$} & \multirow{2}{*}{$\begin{array}{c}\text { N orgânico aplicado } \\
\text { via lodo de esgoto } \\
\text { na quinta aplicação }\end{array}$} & \multicolumn{4}{|c|}{ Lodo de esgoto aplicado } \\
\hline & & & Franca & Barueri & Franca & Barueri \\
\hline & & $\mathrm{mg} \mathrm{kg}^{-1}$ & $\ldots \mathrm{kg} \mathrm{h}$ & $\cdot .^{1(4)}$ & $\ldots \mathrm{n}$ & $\mathrm{kg}^{-1}$ \\
\hline $\begin{array}{l}F 1 N+0 \\
F 1 N+160 \\
F 1 N+320\end{array}$ & $\mathrm{~F}^{(2)} 1 \mathrm{~N}^{(3)}$ & $\begin{array}{r}0 \\
160 \\
320\end{array}$ & $\begin{array}{r}0 \\
6.820 \\
13.640\end{array}$ & $\begin{array}{l}- \\
- \\
-\end{array}$ & $\begin{array}{r}0 \\
3.410 \\
6.820\end{array}$ & $\begin{array}{l}- \\
- \\
-\end{array}$ \\
\hline $\begin{array}{l}\text { F } 2 N+0 \\
\text { F } 2 N+160 \\
F 2 N+320\end{array}$ & $\mathrm{~F} 2 \mathrm{~N}$ & $\begin{array}{r}0 \\
160 \\
320\end{array}$ & $\begin{array}{r}0 \\
6.820 \\
13.640\end{array}$ & $\begin{array}{l}- \\
-\end{array}$ & $\begin{array}{r}0 \\
3.410 \\
6.820\end{array}$ & $\begin{array}{l}- \\
-\end{array}$ \\
\hline $\begin{array}{l}F 4 N+0 \\
F 4 N+640\end{array}$ & $\mathrm{~F} 4 \mathrm{~N}$ & $\begin{array}{r}0 \\
640\end{array}$ & $\begin{array}{r}0 \\
27.280\end{array}$ & - & $\begin{array}{r}0 \\
13.640\end{array}$ & - \\
\hline $\begin{array}{l}\mathrm{F} 8 \mathrm{~N}+0 \\
\mathrm{~F} 8 \mathrm{~N}+1280\end{array}$ & $\mathrm{~F} 8 \mathrm{~N}$ & $\begin{array}{r}0 \\
1.280^{0}\end{array}$ & $\begin{array}{r}0 \\
54.560\end{array}$ & - & $\begin{array}{r}0 \\
27.280\end{array}$ & - \\
\hline $\begin{array}{l}B 1 N+0 \\
B 1 N+160 \\
B 1 N+320\end{array}$ & $\mathrm{~B}^{(2)} 1 \mathrm{~N}$ & $\begin{array}{r}0 \\
160 \\
320\end{array}$ & $\begin{array}{l}- \\
-\end{array}$ & $\begin{array}{r}0 \\
9.100 \\
18.200\end{array}$ & $\begin{array}{l}- \\
- \\
-\end{array}$ & $\begin{array}{r}0 \\
4.550 \\
9.100\end{array}$ \\
\hline $\begin{array}{l}\text { B } 2 \mathrm{~N}+0 \\
\text { B } 2 \mathrm{~N}+160 \\
\text { B } 2 \mathrm{~N}+320\end{array}$ & B $2 \mathrm{~N}$ & $\begin{array}{r}0 \\
160 \\
320\end{array}$ & $\begin{array}{l}- \\
-\end{array}$ & $\begin{array}{r}0 \\
9.100 \\
18.200\end{array}$ & $\begin{array}{l}- \\
- \\
-\end{array}$ & $\begin{array}{r}0 \\
4.550 \\
9.100\end{array}$ \\
\hline $\begin{array}{l}\text { B } 4 N+0 \\
\text { B } 4 N+640 \\
B 8 N+0 \\
\text { B } 8 N+1280\end{array}$ & B $8 \mathrm{~N}$ & $\begin{array}{r}0 \\
640 \\
0 \\
1.280\end{array}$ & $\begin{array}{l}- \\
- \\
-\end{array}$ & $\begin{array}{r}0 \\
36.400 \\
0 \\
72.800\end{array}$ & $\begin{array}{l}- \\
- \\
-\end{array}$ & $\begin{array}{r}0 \\
18.200 \\
0 \\
36.400\end{array}$ \\
\hline
\end{tabular}

(1) Foram feitas quatro aplicações sucessivas, uma em cada cultivo de milho, em experimento de campo. (2) Lodo de esgoto de Franca ou de Barueri, SP. ${ }^{(3)} 1 \mathrm{~N}$ corresponde à aplicação, via lodo de esgoto, da quantidade de $\mathrm{N}$ disponível recomendada para cultivo de milho no Latossolo; $2 \mathrm{~N}, 4 \mathrm{~N}$ e $8 \mathrm{~N}$ representam múltiplos dessa dose. ${ }^{(4)}$ Consideraram-se, para o cálculo, a camada de 0 a $20 \mathrm{~cm}$ de profundidade e densidade do solo igual a $1,0 \mathrm{~kg} \mathrm{dm}^{-3}$.

Quadro 3. Composição química parcial de lodos das Estações de Tratamento de Esgoto de Franca e de Barueri aplicados aos tratamentos no início do experimento de mineralização

\begin{tabular}{|c|c|c|}
\hline \multirow{2}{*}{ Atributo $^{(1)}$} & \multicolumn{2}{|c|}{ Lodo de esgoto } \\
\hline & de Franca & de Barueri \\
\hline Carbono orgânico $\left(\mathrm{g} \mathrm{kg}^{-1}\right)$ & 529,8 & 383,5 \\
\hline pH em água ${ }^{(2)}$ & 6,3 & 6,4 \\
\hline Umidade $(\%)^{(2)}$ & 7,93 & 5,60 \\
\hline N-Kjeldahl (mg kg-1) ${ }^{(2)}$ & 50.264 & 37.193 \\
\hline $\mathrm{N}$-amoniacal $\left(\mathrm{mg} \mathrm{kg}^{-1}\right)^{(2)}$ & 3.320 & 2.008 \\
\hline $\mathrm{N}$ orgânico $(\mathrm{mg} \mathrm{kg} \cdot 1)$ & 46.944 & 35.185 \\
\hline $\mathrm{N}$-nitrato+nitrito $\left(\mathrm{mg} \mathrm{kg}^{-1}\right)^{(2)}$ & 95 & 58 \\
\hline
\end{tabular}

(1) Análises segundo Tedesco et al. (1995). ${ }^{(2)}$ Análises em amostras secas ao ar; resultados expressos com base em massa de matéria seca. orgânicas foi calculada pela diferença entre as quantidades de $\mathrm{N}$ mineralizadas em 105 dias nos tratamentos com e sem aplicação de lodo, dividida pela quantidade de N orgânico aplicada via lodo de esgoto. A estimativa da fração de mineralização potencial foi obtida pela diferença entre as quantidades de $\mathrm{N}$ potencialmente mineralizáveis nos tratamentos com e sem aplicação de lodo (estimadas pelo modelo matemático), divididas pela quantidade de $\mathrm{N}$ orgânico aplicada ao tratamento via lodo de esgoto.

\section{RESULTADOS E DISCUSSÃO}

Os teores de $\mathrm{N}-\mathrm{NH}_{4}{ }^{+}$, de $\mathrm{N}-\left(\mathrm{NO}_{3}{ }^{-}+\mathrm{NO}_{2}{ }^{-}\right)$, de $\mathrm{N}$ mineral acumulado e os valores de $\mathrm{pH}$ medidos durante a incubação encontram-se na figura 1 para os tratamentos com o lodo de Franca e na figura 2 para os tratamentos com o lodo de Barueri. 
Os lodos de esgoto utilizados no experimento continham elevados teores de $\mathrm{N}$ amoniacal e baixos teores de N nítrico (Quadro 3). Por essa razão, houve diferenças significativas entre os tratamentos com e sem aplicação dos resíduos no início do experimento. Aos 14 dias, os teores encontrados estabilizaram-se em valores baixos (o valor máximo observado foi de $11,7 \mathrm{mg} \mathrm{kg}^{-1}$ ). No decorrer da incubação, observaram-
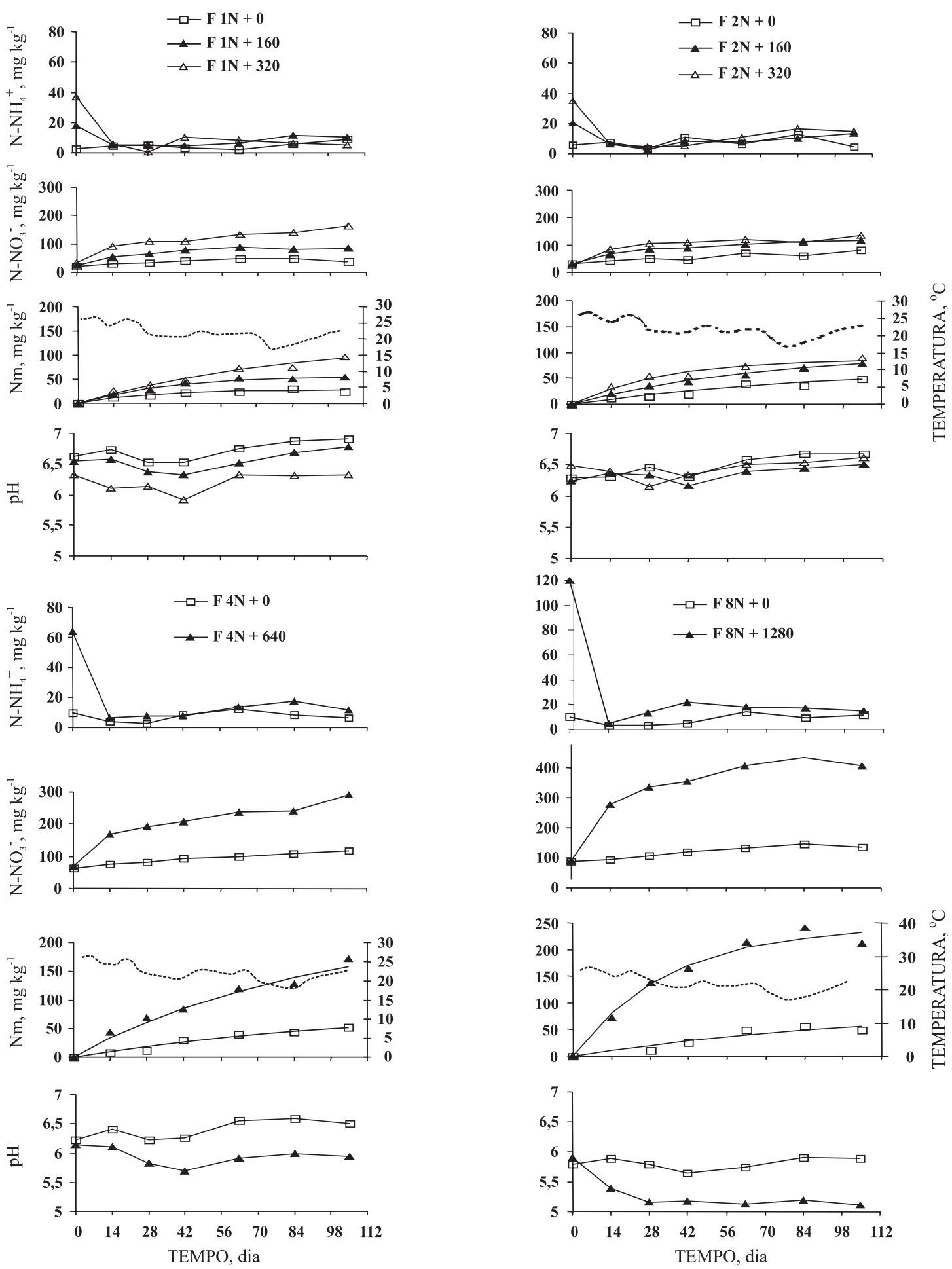

Figura 1. Efeito da quinta aplicação de doses de $\mathrm{N}$ orgânico $\left(0,160,320,640\right.$ ou $\left.1.280 \mathrm{mg} \mathrm{kg}^{-1}\right)$, via lodo de esgoto de Franca, sobre os teores de $\mathrm{N}$ amoniacal e nítrico, $\mathrm{N}$ mineralizado (Nm) e pH, durante incubação de Latossolo pré-tratado com o resíduo. F $1 \mathrm{~N}$ representa o Latossolo pré-tratado, em quatro cultivos sucessivos, com a dose de lodo calculada para adubação nitrogenada de milho; F 2N, F 4N e F 8N representam seus múltiplos. 
se poucas variações significativas no teor de $\mathrm{N}-\mathrm{NH}_{4}^{+}$. Aos 28 dias, houve diferenças significativas entre os tratamentos F $8 \mathrm{~N}+0$ e F $8 \mathrm{~N}+1280$, entre B $4 \mathrm{~N}+0$ e B $4 \mathrm{~N}+640$, e entre B $8 \mathrm{~N}+0$ e B $8 \mathrm{~N}+1280$. Aos 84 dias, a quinta aplicação do lodo de Barueri afetou significativamente o teor de $\mathrm{N}-\mathrm{NH}_{4}{ }^{+}$nos tratamentos
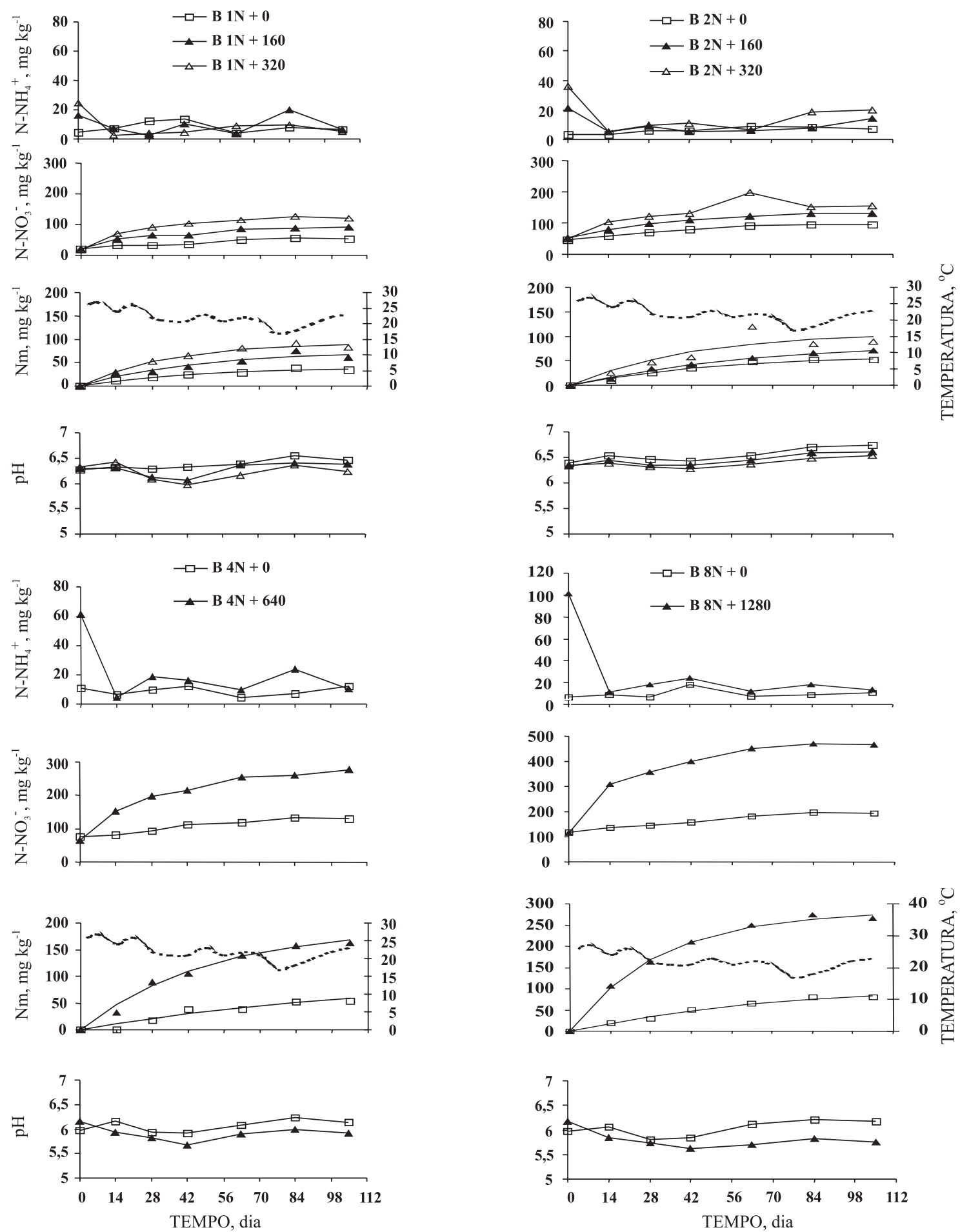

Figura 2. Efeito da quinta aplicação de doses de $\mathrm{N}$ orgânico $\left(0,160,320,640\right.$ ou $\left.1.280 \mathrm{mg} \mathrm{kg}^{-1}\right)$ via lodo de esgoto de Barueri, sobre os teores de $\mathrm{N}$ amoniacal e $\mathrm{N}$-nítrico, $\mathrm{N}$ mineralizado (Nm) e pH, durante incubação de Latossolo pré-tratado com o resíduo. B $1 \mathrm{~N}$ representa o Latossolo pré-tratado, em quatro cultivos sucessivos, com a dose de lodo calculada para adubação nitrogenada de milho; B $2 \mathrm{~N}$, B 4N e B 8N representam seus múltiplos. 
B $8 \mathrm{~N}+1280, \mathrm{~B} 4 \mathrm{~N}+640, \mathrm{~B} 2 \mathrm{~N}+320$ e B $1 \mathrm{~N}+160$. Aos 105 dias, não houve diferenças significativas para o teor de $\mathrm{N}_{-} \mathrm{NH}_{4}{ }^{+}$, que se encontrava baixo em todos os tratamentos (o valor máximo observado foi de $19,7 \mathrm{mg} \mathrm{kg}^{-1}$ ). Esses resultados indicam que praticamente todo o amônio gerado durante a decomposição orgânica dos compostos nitrogenados foi rapidamente convertido em nitrato, sem se acumular em grande quantidade no solo. Os teores de $\mathrm{N}^{-\mathrm{NO}_{3}}$, baixos no início da incubação, aumentaram significativamente nos tratamentos com aplicação do lodo, em todas as épocas de avaliação (Figuras 1 e 2). Também houve aumento linear significativo ao longo da incubação em todos os tratamentos. No final da incubação, o teor de $\mathrm{N}_{-} \mathrm{NO}_{3}{ }^{-}$foi maior quando a dose aplicada do lodo de esgoto foi maior.

Em geral, a taxa de mineralização de lodo de esgoto aplicado a solos é maior no início da incubação e decresce com o tempo (Parker \& Sommers, 1983; Lindemann \& Cardenas, 1984; Banerjee et al., 1997), ocorrendo aumento no teor de nitrato à medida que 0 teor de amônio diminui. Assim, Boeira et al. (2002), em laboratório, e Vieira \& Cardoso (2003) e Tarrasón et al. (2008), em campo, observaram que o amônio permaneceu por mais tempo em teores mais altos ao aplicarem lodo de esgoto em solos sem aplicações prévias do resíduo. Neste trabalho, houve diferenças nesse comportamento em todos os tratamentos (Figuras 1 e 2). Rápido decréscimo nos teores de N$\mathrm{NH}_{4}{ }^{+}$, entre seis e 27 dias, também foi observado por Vieira et al. (2005) em campo, após a terceira aplicação de lodo de Barueri, nesse mesmo Latossolo. Essa diferença, entre solos previamente tratados ou não com lodo de esgoto, pode ser devida à adaptação da população microbiana do solo às alterações causadas pelas aplicações prévias, resultando em maior eficiência do processo de nitrificação, ou ativação de outros processos biológicos e bioquímicos. A origem do lodo de esgoto não parece ter afetado a mineralização, mesmo sendo de Barueri o mais rico em metais pesados (Boeira et al., 2002). Avaliando a biomassa microbiana nesse Latossolo, Fernandes et al. (2005) não observaram efeitos prejudiciais do lodo de Barueri em relação ao solo não tratado, ou tratado com fertilização mineral. Os autores consideraram a hipótese de que a matéria orgânica do lodo tenha estimulado a atividade dos microrganismos do solo.

A avaliação do $\mathrm{pH}$ ao longo da incubação (Figuras 1 e 2) mostrou aumento linear significativo em alguns tratamentos, mas não em outros. Esses resultados podem ser atribuídos ao efeito residual da calagem efetuada seis meses antes, pois a nitrificação, que é um processo acidificante (Yan et al., 1996), ocorreu de forma linear crescente em todos os tratamentos. Somente no tratamento F $8 \mathrm{~N}+1.280$ houve decréscimo significativo de $\mathrm{pH}$ no período estudado, evidenciando o poder de acidificação do lodo de esgoto de Franca, também observado por Boeira et al. (2002).
Os dados de $\mathrm{N}$ líquido mineralizado encontram-se nas figuras 1 e 2, nos gráficos Nm em função do tempo, que incluem os dados relativos à temperatura ambiente durante a incubação. Em geral, aos 105 dias houve o maior acúmulo de N mineralizado. Em $35 \%$ dos tratamentos, os maiores valores acumulados foram aos 84 dias, e no tratamento B $2 \mathrm{~N}+320$ foi aos 63 dias, com posterior decréscimo da variável, indicando perdas ou imobilização de N. O decréscimo na temperatura ambiente pode ter contribuído para esse resultado.

Os valores calculados aos 105 dias para a fração de mineralização do $\mathrm{N}$ de compostos orgânicos (FMN) encontram-se no quadro 4, com os respectivos intervalos de confiança a $95 \%$. Houve sobreposição dos valores dos intervalos de confiança em todos os tratamentos. Dessa forma, não foram obtidas evidências de efeito significativo de aplicações prévias sobre a FMN do lodo de esgoto recentemente aplicado. E tem-se confiança de $95 \%$ de que os valores da FMN, calculada experimentalmente na quinta aplicação, encontram-se entre 7 e $27 \%$. Resultados independentes do histórico da área sobre a mineralização de lodo recentemente aplicado também foram obtidos por outros autores. Lindemann et al. (1988) aplicaram dose equivalente a $60.000 \mathrm{~kg} \mathrm{ha}^{-1} \mathrm{em}$ solos tratados um ano antes, e $67.300 \mathrm{~kg} \mathrm{ha}^{-1} \mathrm{em}$ solos tratados dois anos antes, e não obtiveram efeitos sobre a FMN do lodo. Gilmour et al. (1996), avaliando áreas com disposição anual de 0, 647.000 e $418.000 \mathrm{~kg} \mathrm{ha}^{-1}$ de lodo de esgoto, durante sete anos, não observaram diferenças na FMN. Wiseman \& Zibilske (1988), que compararam a aplicação de $13.300 \mathrm{~kg} \mathrm{ha}^{-1}$ de lodo doméstico em solo não tratado ou em solo tratado 40 dias antes com essa mesma quantidade de lodo, relataram que houve pouco efeito da aplicação inicial sobre a mineralização dos compostos nitrogenados na aplicação seguinte.

As quantidades acumuladas de $\mathrm{N}$ mineral $(\mathrm{Nm})$ geradas durante a incubação foram proporcionais às quantidades de $\mathrm{N}$ orgânico do lodo aplicado. A associação significativa entre essas variáveis $(r=0,71$, $\mathrm{p}<0,01$ ) permitiu obterem-se os parâmetros do modelo exponencial simples apresentados no quadro 4 . Nesse modelo, identifica-se o reservatório de $\mathrm{N}$ mineralizável no resíduo estudado, o qual, teoricamente, tem um tamanho definido e é independente das condições ambientais ou do procedimento usado para o ajuste dos dados (Cabrera et al., 2005). Esse parâmetro é o potencial de mineralização do lodo de esgoto, com o que se pode estimar a fração do $\mathrm{N}$ orgânico no resíduo que será mineralizada durante o ciclo da cultura (CETESB, 1999). Somando-se esta parcela de $\mathrm{N}$ às formas minerais (amônio + nitrato) contidas no lodo, obtém-se a quantidade de $\mathrm{N}$ disponível do lodo de esgoto. Esse índice é utilizado para a definição da dose do resíduo a ser usada em solos agrícolas. Porém, em reaplicações de lodo de esgoto, deve-se considerar que a quantidade de $\mathrm{N}$ disponível no solo será maior que em solos não tratados, pois há teores residuais de $\mathrm{N}$ mineral e de matéria orgânica. Neste trabalho, 
Quadro 4. Relação C:N, N orgânico, fração de mineralização de N orgânico (FMN) calculada para os lodo de esgoto, parâmetros estimados para descrição da mineralização de $\mathbf{N}$ segundo o modelo exponencial simples e FMN estimada nos tratamentos após 105 dias de incubação

\begin{tabular}{|c|c|c|c|c|c|c|c|c|c|}
\hline \multirow{2}{*}{ Tratamento } & \multirow{2}{*}{$\begin{array}{c}\mathrm{C}: \mathrm{N} \\
(0 \text { dias })\end{array}$} & \multirow{2}{*}{$\begin{array}{l}\text { N orgânico } \\
\text { (105 dias) }\end{array}$} & \multirow{2}{*}{$\begin{array}{c}\text { FMN } \\
\text { calculada }\end{array}$} & \multicolumn{5}{|c|}{$\operatorname{Nm}=\operatorname{No}\left(1-e^{-k t}\right)$} & \multirow{2}{*}{$\begin{array}{c}\text { FMN } \\
\text { estimada }\end{array}$} \\
\hline & & & & $\operatorname{IC}(95 \%)^{(2)}$ & No ${ }^{(3)}$ & $\mathbf{k}^{(4)}$ & $\mathbf{t}^{1 / 2}$ & $\mathrm{R}^{2(6)}$ & \\
\hline & & $\mathrm{mg} \mathrm{kg-1}$ & - $\%$ & - & $\mathrm{mg} \mathrm{kg}^{-1}$ & $\operatorname{dia}^{-1}$ & dia & 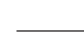 & $\%$ \\
\hline $\mathrm{F} 1 \mathrm{~N}+0$ & 11,1 & $1.068 \mathrm{~b}^{(1)}$ & - & & 28 & 0,037 & 19 & 86 & - \\
\hline $\mathrm{F} 1 \mathrm{~N}+160$ & 11,2 & $1.229 \mathrm{~b}$ & 18 & $(14-23)$ & 58 & 0,028 & 25 & 95 & 19 \\
\hline $\mathrm{F} 1 \mathrm{~N}+320$ & 11,2 & $1.598 \mathrm{a}$ & 23 & $(18-27)$ & 125 & 0,013 & 53 & 95 & 30 \\
\hline $\mathrm{F} 2 \mathrm{~N}+0$ & 9,6 & $1.227 \mathrm{a}$ & & & 70 & 0,011 & 63 & 81 & - \\
\hline $\mathrm{F} 2 \mathrm{~N}+160$ & 9,8 & $1.304 \mathrm{a}$ & 18 & $(14-23)$ & 98 & 0,015 & 46 & 92 & 18 \\
\hline $\mathrm{F} 2 \mathrm{~N}+320$ & 9,9 & $1.358 \mathrm{a}$ & 13 & $(8-17)$ & 88 & 0,030 & 23 & 92 & 6 \\
\hline $\mathrm{F} 4 \mathrm{~N}+0$ & 9,9 & $1.524 \mathrm{a}$ & - & & 93 & 0,008 & 87 & 95 & - \\
\hline $\mathrm{F} 4 \mathrm{~N}+640$ & 10,4 & $1.913 \mathrm{a}$ & 19 & $(14-23)$ & 230 & 0,011 & 63 & 88 & 21 \\
\hline $\mathrm{F} 8 \mathrm{~N}+0$ & 6,9 & $2.172 \mathrm{~b}$ & - & & 100 & 0,008 & 87 & 75 & - \\
\hline $\mathrm{F} 8 \mathrm{~N}+1280$ & 8,5 & $3.264 \mathrm{a}$ & 13 & $(8-17)$ & 243 & 0,029 & 24 & 86 & 11 \\
\hline $\mathrm{B} 1 \mathrm{~N}+0$ & 10,2 & $1.157 \mathrm{a}$ & - & & 40 & 0,022 & 32 & 88 & - \\
\hline B $1 N+160$ & 10,2 & $1.236 \mathrm{a}$ & 17 & $(13-22)$ & 75 & 0,022 & 32 & 92 & 22 \\
\hline $\mathrm{B} 1 \mathrm{~N}+320$ & 10,3 & $1.265 \mathrm{a}$ & 15 & $(11-20)$ & 93 & 0,029 & 24 & 97 & 17 \\
\hline B $2 N+0$ & 10,0 & $1.265 \mathrm{~b}$ & - & & 63 & 0,019 & 36 & 92 & - \\
\hline B $2 N+160$ & 10,1 & $1.434 \mathrm{ab}$ & 12 & $(8-17)$ & 90 & 0,015 & 46 & 97 & 17 \\
\hline B $2 N+320$ & 10,1 & $1.541 \mathrm{a}$ & 12 & $(7-16)$ & 108 & 0,024 & 29 & 63 & 14 \\
\hline $\mathrm{B} 4 \mathrm{~N}+0$ & 8,6 & $1.835 \mathrm{a}$ & - & & 95 & 0,009 & 77 & 66 & - \\
\hline B $4 N+640$ & 9,3 & $2.234 \mathrm{a}$ & 17 & $(12-21)$ & 192 & 0,020 & 35 & 97 & 15 \\
\hline $\mathrm{B} 8 \mathrm{~N}+0$ & 7,2 & $2.321 \mathrm{a}$ & - & & 113 & 0,013 & 53 & 92 & - \\
\hline B $8 N+1280$ & 8,5 & $3.358 \mathrm{a}$ & 14 & $(10-19)$ & 284 & 0,032 & 22 & 97 & 13 \\
\hline
\end{tabular}

${ }^{(1)}$ Médias seguidas pela mesma letra na coluna não diferem entre si pelo teste de Tukey $(p<0,01)$, em cada dose prévia de lodo de esgoto. ${ }^{(2)}$ Intervalo de confiança $(\mathrm{p} \leq 0,05) .{ }^{(3)} \mathrm{N}$ potencialmente mineralizável. ${ }^{(4)}$ Constante da taxa de mineralização. ${ }^{(5)}$ Meiavida: tempo necessário para mineralização de $50 \%$ do $\mathrm{N}$ potencialmente mineralizável; $\mathrm{t} 1 \frac{12}{2}(\ln 2) / \mathrm{k}$. ${ }^{(6)}$ Coeficiente de determinação da estimativa de $\mathrm{Nm}$ : indica a porcentagem da variabilidade em $\mathrm{Nm}$, que é explicada pelo modelo ajustado.

encontraram-se teores residuais de 24 a $124 \mathrm{mg} \mathrm{kg}^{-1}$ de $\mathrm{N}$ mineral nos tratamentos sem a quinta aplicação de lodo, no início da incubação (Figuras 1 e 2). Durante a incubação dos tratamentos, houve geração de $\mathrm{N}$ mineral em quantidades superiores às necessárias em um cultivo anual exigente em $\mathrm{N}$, com exceção de $\mathrm{F}$ $1 \mathrm{~N}+0$ e B $1 \mathrm{~N}+0$ (No, Quadro 4), potencializando o acúmulo do nutriente em formas minerais suscetíveis à lixiviação e, ou, a outros processos relativos à dinâmica de N. No final da incubação, as diferenças no acúmulo de $\mathrm{N}$ orgânico chegaram a $50 \%$ entre os tratamentos $\mathrm{F} 1 \mathrm{~N}+320$ e $\mathrm{F} 1 \mathrm{~N}+0$ e entre $\mathrm{F}$ $8 \mathrm{~N}+1280$ e F 8N + 0 (Quadro 4), o que também foi observado em experimentos em campo (Oliveira et al., 2001; Boeira \& Souza, 2007). Os resultados mostram que esses efeitos residuais devem ser introduzidos no cálculo das doses de lodo de esgoto para aplicações repetidas na mesma área.

O potencial de mineralização do lodo de esgoto foi estimado pela diferença entre os potenciais de mineralização nos tratamentos com e sem a quinta aplicação do lodo. Como exemplo, verifica-se, no quadro 4, que as taxas de mineralização estimadas (No) nos tratamentos $\mathrm{F} 1 \mathrm{~N}+160$ e $\mathrm{F} 1 \mathrm{~N}+0$ foram de 58 e $28 \mathrm{mg} \mathrm{kg}^{-1}$, respectivamente. Por diferença, verifica-se que a mineralização potencial estimada para o lodo de esgoto de Franca foi de $30 \mathrm{mg} \mathrm{kg}^{-1} \mathrm{de}$ N. Aplicando-se a equação do modelo exponencial simples para $\mathrm{t}=14$ dias (Quadro 4), obtêm-se valores de $\mathrm{Nm}$ de 18,8 e 11,3 mg kg-1 para os tratamentos $\mathrm{F}$ $1 \mathrm{~N}+160$ e $\mathrm{F} 1 \mathrm{~N}+0$, respectivamente. Então, por diferença, estimou-se que $7,5 \mathrm{mg} \mathrm{kg}^{-1}$ do $\mathrm{N}$ orgânico aplicado ao solo seriam mineralizados nas duas semanas iniciais após a aplicação, o que representa $25 \%$ do total a ser mineralizado no ano agrícola $(7,5 /$ $30 \mathrm{mg} \mathrm{kg}^{-1}$ ). Avaliando-se todos os tratamentos de forma similar, nos primeiros 14 dias de incubação, estimaram-se valores entre 10 e $49 \%$ do total a ser mineralizado, com liberações equivalentes a $140 \mathrm{~kg} \mathrm{ha}^{-1}$ de $\mathrm{N}$ no tratamento F $8 \mathrm{~N}+1280$, e de $168 \mathrm{~kg} \mathrm{ha}^{-1}$ de $\mathrm{N}$ no tratamento B $8 \mathrm{~N}+1280$. Em campo, Vieira et al. (2005) verificaram que entre 7 e 22 \% do N orgânico do lodo de Barueri foi mineralizado em apenas seis dias após a terceira aplicação anual nesse Latossolo. 
Estes resultados confirmam a elevada taxa inicial de mineralização do lodo, em condições adequadas de temperatura e de umidade, tanto em laboratório quanto no campo, liberando a maior parte do $\mathrm{N}$ disponível em época em que as plantas ainda não germinaram ou recém-germinadas.

A intensa mineralização inicial dos resíduos somada à contribuição dos teores de $\mathrm{N}$ mineral nos lodos $(0,2-0,3 \%$, conforme Quadro 3; Figuras 1 e 2, teores de $\mathrm{N}-\mathrm{NH}_{4}{ }^{+}$no dia), somadas aos efeitos residuais das aplicações anteriores (Figuras 1 e 2, teores de $\mathrm{N}-\mathrm{NO}_{3}{ }^{-}$crescentes nos tratamentos sem a quinta aplicação de lodos), concorreram para que houvesse grande disponibilidade de $\mathrm{N}$, em todos os tratamentos, no período inicial da incubação. Após 14 dias de incubação, a quantidade de $\mathrm{N}$ disponível medida variou de $36 \mathrm{mg} \mathrm{kg}^{-1}$ no tratamento $\mathrm{F} 1 \mathrm{~N}+0$ a $320 \mathrm{mg} \mathrm{kg}^{-1}$ no tratamento B $8 \mathrm{~N}+1.280$. Deve-se salientar, ainda, que o lodo de esgoto é aplicado com elevado teor de água (> $350 \%$, base seca), e que o lodo utilizado neste trabalho continha teor de água muito baixo (em torno de $7 \%$, Quadro 3) para permitir homogeneização adequada com o solo durante o preparo dos tratamentos no laboratório. Como a secagem reduziu o teor de $\mathrm{N}$ amoniacal do lodo em cerca de um terço, a disponibilidade inicial de $\mathrm{N}$ nítrico em campo pode então vir a ser mais intensa ainda do que foi observada em laboratório. Este é um grave problema da aplicação de lodo de esgoto por incorporação ao solo antes do plantio, quando ainda não há demanda pelas plantas, gerando grandes perdas de N por lixiviação (Dynia et al., 2006).

A partir dos valores do potencial de mineralização obtidos nos tratamentos, estimou-se entre 6 e $30 \%$ a FMN dos dois tipos de lodo de esgoto (Quadro 4). Boeira et al. (2002) estimaram entre 20 e $38 \%$ a FMN na primeira aplicação do lodo de esgoto no mesmo Latossolo deste trabalho; Lindemann \& Cardenas (1984) obtiveram taxas de mineralização de 56 a 72 \%; Lerch et al. (1992) encontraram variação de 27 a $55 \%$; Barretto (1995) obteve valores entre 24 e $35 \%$; Oliveira (1995) obteve $47 \%$. O índice FMN, normalmente obtido em laboratório com amplas faixas de variação, é útil para o cálculo de taxas de resíduo a aplicar (CETESB, 1999; USEPA, 1996), e pode ser validado em trabalhos de campo.

Considerando-se o manejo prévio do Latossolo aqui estudado, Vieira \& Cardoso (2003) e Vieira et al. (2005) relatam que houve intensa nitrificação, com lixiviação de $\mathrm{N}$ abaixo de $20 \mathrm{~cm}$, mesmo nas menores taxas aplicadas de lodo de Barueri, durante os três primeiros cultivos de milho. Avaliando os teores de nitrato até a profundidade de 3,0 m, Dynia et al. (2006) observaram que, após quatro aplicações anuais do lodo de Barueri (6.000 kg ha-1 ano $^{-1}$ ) e do lodo de Franca (3.600 kg ha ${ }^{-1}$ ano $\left.^{-1}\right)$, o Latossolo atingiu seu limite de carga, o que resultou em risco de contaminação do lençol freático. Esses resultados indicam que as quantidades aplicadas foram excessivas. Em parte, pode-se supor que a fração de mineralização utilizada foi subestimada em relação à mineralização ocorrida no campo, como sugerido por Vieira \& Cardoso (2003) e Vieira et al. (2005). Além disso, deve-se também considerar que os efeitos residuais das aplicações anteriores do lodo de esgoto não foram avaliados para os cálculos das quantidades a aplicar. Barbarick et al. (1996) também concluíram que pode haver subestimação da geração de $\mathrm{N}$ mineral utilizando-se o porcentual de $20 \%$ sugerido por USEPA (1996) para a FMN de lodo de esgoto de digestão anaeróbia, pois a mineralização em campo após aplicações sucessivas mostrou-se muito mais intensa.

Para o solo e lodo de esgoto deste estudo, considerando-se reaplicações dos resíduos pela incorporação no solo, os resultados obtidos indicam que, preventivamente, a FMN mínima a ser utilizada deve ser de $30 \%$, que foi o maior valor estimado experimentalmente, levando-se em conta o grande potencial de riscos à saúde pública desse material (CONAMA, 2006). Porém, para determinar a dose subsequente a outras aplicações, outros fatores além do $\mathrm{N}$ disponível no lodo de esgoto e da necessidade da planta devem ser considerados, entre eles o efeito residual das aplicações anteriores, incluindo-se a quantidade de $\mathrm{N}$ potencialmente mineralizável da camada arável, o teor de $\mathrm{N}$ mineral na camada de exploração de raízes e o monitoramento dos teores de nitrato no perfil do solo, bem como a inexistência de sincronismo entre a época de maior liberação de $\mathrm{N}$ mineral pelo lodo e a época de demanda das plantas.

\section{CONCLUSÔES}

1. A fração de mineralização de $\mathrm{N}$ de compostos orgânicos do lodo de esgoto, na quinta aplicação, alcançou 30\%, e foi independente das quantidades previamente aplicadas ao Latossolo.

2. A origem do lodo de esgoto, urbana ou urbanoindustrial, não afetou sua mineralização.

3. Em solos manejados com incorporações sucessivas de lodo de esgoto, as normas atuais devem agregar índices que considerem o efeito residual de $\mathrm{N}$ e a época de aplicação para o cálculo da dose agronômica das reaplicações.

\section{LITERATURA CITADA}

BANERJEE, M.R.; BURTON, D.L. \& DEPOE, S. Impact of sewage sludge application on soil biological characteristics. Agric. Ecosyst. Environ., 66:241-249, 1997.

BARBARICK, K.A.; IPPOLITO, J.A. \& WESTFALL, D.G. Distribution and mineralization of biosolids nitrogen applied to dryland wheat. J. Environ. Qual., 25:796-801, 1996.

BARRETTO, M.C.V. Degradação da fração orgânica de resíduos e efeitos em algumas propriedades químicas e físicas de dois solos. Piracicaba, Escola Superior de Agricultura Luiz de Queiroz, 1995. (Tese de Doutorado) 
BOEIRA, R.C.; LIGO, M.A.V. \& DYNIA, J.F. Mineralização de nitrogênio em solo tropical tratado com lodo de esgoto. Pesq. Agropec. Bras., 37:1639-1647, 2002.

BOEIRA, R.C. \& SOUZA, M.D. Estoques de carbono orgânico e de nitrogênio, $\mathrm{pH}$ e densidade de um Latossolo após três aplicações de lodo de esgoto. R. Bras. Ci. Solo., 31:581590, 2007.

BOYD, G.R.; REEMTSMA, H.; GRIMM, D.A. \& MITRA, S. Pharmaceuticals and personal care products (PPCPs) in surface and treated waters of Louisiana, USA and Ontario, Canada. Sci. Total Environ., 311:135-149, 2003.

CABRERA, M.L.; KISSEL, D.E. \& VIGIL, M.F. Nitrogen mineralization from organic residues: Research opportunities. J. Environ. Qual., 34:75-79, 2005.

CAMARGO, O.A.; MONIZ, A.C.; JORGE, J.A. \& VALADARES, J.M.A.S. Métodos de análise química, mineralógica e física de solos do Instituto Agronômico de Campinas. Campinas, Instituto Agronômico de Campinas, 1986. 95p. (IAC. Boletim Técnico, 106).

CARBALLA, M.; OMIL, F.; LEMA, J.M.; LOMPART, M.; GARCIA, C.; RODRIGUES, I.; GOMEZ, M. \& TERNES, T. Behaviour of pharmaceuticals and personal care products in a sewage treatment plant of northwest Spain. Water Sci. Technol., 52:29-35, 2005.

CETESB. Aplicação de lodos de sistemas de tratamento biológico em áreas agrícolas: Critérios para projeto e operação. São Paulo, 1999. 32p. (Manual Técnico, 230)

CONSELHO NACIONAL DO MEIO AMBIENTE - CONAMA. Resolução n. 375 , de 29 de agosto de 2006. Disponível em: $<$ http://www.ecolnews.com.br/legislacao/resolucoes/ res_indice.htm>. Acesso em 15 jan. de 2007.

COUNCIL OF THE EUROPEAN COMMUNITIES. CEC. Council Directive of 12 December 1991 concerning the protection of waters against pollution caused by nitrates from agricultural sources (91/676/EEC). Official J. Europ. Comm., 375:1-8, 1991.

DYNIA, J.F.; SOUZA, M.D. \& BOEIRA, R.C. Lixiviação de nitrato em Latossolo cultivado com milho após aplicações sucessivas de lodo de esgoto. Pesq. Agropec. Bras., 41:855862,2006

ESTADOS UNIDOS. Environmental Protection Agency USEPA. Standards for the use and disposal of sewage sludge. Washington, 1996. (Code of Federal Regulations 40 Part 503).

FERNANDES, S.A.P.; BETTIOL, W. \& CERRI, C.C. Effect of sewage sludge on microbial biomass, basal respiration, metabolic quotient and soil enzymatic activity. Appl. Soil Ecol., 30:65-77, 2005.

FORSTE, J.B. Land application. In: GIROVICH, M.J., ed. Biosolids treatment and management - Processes for beneficial use. New York, Marcel Dekker, 1996. p.389-448.

GARAU, M.A.; FELIPÓ, M.T. \& VILLA, M.C.R.D. Nitrogen mineralization of sewage sludges in soils. J. Environ. Qual., 15:225-228, 1986.

GILMOUR, J.T.; ROMAN, F. \& CLARK, M.D. Decomposition of biosolids in a disposal site soil. J. Environ. Qual., 25:10831086, 1996
HETTENBACK, T.; COHEN, B.; WILES, R. \& COOK, K. Dumping sewage sludge on organic farms? Why USDA should just say no. Washington, USDA Environmental Working Group, 1998. 9p.

LERCH, R.N.; BARBARICK, K.A.; SOMMERS, L.E. \& WESTFALL, D.G. Sewage sludge proteins as labile carbon and nitrogen sources. Soil Sci. Soc. Am. J., 56:1470$1476,1992$.

LINDEMANN, W.C. \& CARDENAS, M. Nitrogen mineralization potential and nitrogen transformations of sludgeamended soil. Soil Sci. Soc. Am. J., 48:1072-1077, 1984.

LINDEMANN, W.C.; CONNELL, G. \& URQUHART, N.S. Previous sludge addition effects on nitrogen mineralization in freshly amended soil. Soil Sci. Soc. Am. J., 52:109-112, 1988.

OLIVEIRA, F.C. Metais pesados e formas nitrogenadas em solos tratados com lodo de esgoto. Piracicaba, Escola Superior de Agricultura Luiz de Queiroz, 1995. (Tese de Mestrado)

OLIVEIRA, F.C.; MATTIAZZO, M.E.; MARCIANO, C.R. \& MORAES, S.O. Lixiviação de nitrato em um Latossolo Amarelo distrófico tratado com lodo de esgoto e cultivado com cana-de-açúcar. Sci. Agric., 58:171-180, 2001.

PARAÍBA, L.C.; BOEIRA, R.C.; JONSSON, C.M. \& CARRASCO, J.M. Fator de bioconcentração de poluentes orgânicos de lodos em frutos de laranjeiras. Pesticidas: R. Ecotoxicol. Meio Amb., 16:125-134, 2006.

PARKER, C.F. \& SOMMERS, L.E. Mineralization of nitrogen in sewage sludges. J. Environ. Qual., 12:150-156, 1983.

PIMENTEL, D. Green revolution agriculture and chemical hazards. Sci. Total Environ., 188:586-598, 1996.

PRATT, P.F.; BROADBENT, F.E. \& MARTIN, J.P. Using organic wastes as nitrogen fertilizer. California Agric., 27:10-13, 1973

REINO UNIDO. The protection of water against agricultural nitrate pollution (England and Wales): Regulations 1996. London, HMSO, 1996. (Statutory Instrument, 888)

SAITO, M.L. O uso do lodo de esgoto na agricultura: Precauções com os contaminantes orgânicos. Jaguariúna, Embrapa Meio Ambiente, 2007. 36p. (Embrapa Meio Ambiente. Documentos, 64) Disponível em: <http:// www.cnpma.embrapa.br/download/documentos_64.pdf>.

SCHRODER, J.L.; ZHANGA, H.; ZHOUB, D.; BASTAC, N.; RAUNA, W.R.; PAYTOND, M.E. \& ZAZULAKE, A. The effect of long-term annual application of biosolids on soil properties, phosphorus, and metals. Soil Sci. Soc. Am. J., $72: 73-82,2008$

SHOBER, A.L. \& SIMS, J.T. Phosphorus restrictions for land application of biosolids: Current status and future trends. J. Environ. Qual., 32:1955-1964, 2003.

SMITH, J.L.; SCHNABEL, R.R.; MCNEAL, B.L. \& CAMPBELL, G.S. Potential errors in the first-order model for estimating soil nitrogen mineralization potentials. Soil Sci. Soc. Am. J., 44:996-1000, 1980.

STANFORD, G. \& SMITH, S.J. Nitrogen mineralization potentials of soils. Soil Sci. Soc. Am. J., 36:465-471, 1972. 
TARRASÓN, D.; OJEDA, G.; ORTIZ, O. \& ALCAÑIZ, J.M. Differences on nitrogen availability in a soil amended with fresh, composted and thermally-dried sewage sludge. Biores. Technol., 99:252-259, 2008.

TEDESCO, M.J.; GIANELLO, C.; BISSANI, C.A.; BOHNEN, H. \& VOKWEISS, S.J. Análise de solo, plantas e outros materiais. 2.ed. Porto Alegre, Universidade Federal do Rio Grande do Sul, 1995. 174p. (Boletim Técnico, 5)

TERNES, T.A. Occurrence of drugs in German sewage treatment plants and rivers. Water Res., 32:3245-3260, 1998.

TERNES, T.A.; STUMPF, M.; MUELLER, J.; HABERER, K.; WILKEN, R. D. \& SERVOS, M. Beharvior and occurrence of estrogens in municipal sewage treatment plants: I. Investigations in Germany, Canada and Brazil. Sci. Total Environ., 225:81-80, 1999.

TSUTIYA, M.T. Alternativas de disposição final de biossólidos gerados em estações de tratamento de esgotos. In: BETTIOL, W. \& CAMARGO, O.A., eds. Impacto ambiental do uso agrícola do lodo de esgoto. Jaguariúna, Embrapa Meio Ambiente, 2000. p.69-105.
TSUTIYA, M.T. Características de biossólidos gerados em estações de tratamento de esgotos. In: TSUTIYA, M.T.; COMPARINI, J.B.; SOBRINHO, A.P.; HESPANOL, I.; CARVALHO, P.C.T. \& MELFI, A.J., eds. Biossólidos na agricultura. São Paulo, SABESP, 2001. p.89-131.

VIEIRA, R.F. \& CARDOSO, A.A. Variações nos teores de nitrogênio mineral em solo suplementado com lodo de esgoto. Pesq. Agropec. Bras., 38:867-874, 2003.

VIEIRA, R.F.; MAIA, A.H.N. \& TEIXEIRA, M.A. Inorganic nitrogen in a tropical soil with frequent amendments of sewage sludge. Biol. Fert. Soils, 41:273-279, 2005.

WALLACE, A. \& WALLACE, G.A. A possible flaw in EPA's 1993 new sludge rule due heavy metal interactions. Comm. Soil Sci. Plant Anal., 25:129-135, 1994.

WISEMAN, J.T. \& ZIBILSKE, L.M. Effects of sludge application sequence on carbon and nitrogen mineralization in soil. J. Environ. Qual., 17, 334-339, 1988.

YAN, F.; SCHUBERT, S. \& MENGEL, K. Soil pH increase due to biological decarboxylation of organic anions. Soil Biol. Biochem., 28:617-624, 1996. 